\title{
Focused ion beam modification of atomic force microscopy tips for near-field scanning optical microscopy
}

\author{
Jeffrey R. Krogmeier and Robert C. Dunn ${ }^{\text {a) }}$ \\ Department of Chemistry, University of Kansas, Malott Hall, Lawrence, Kansas 66045
}

(Received 27 August 2001; accepted for publication 2 November 2001)

\begin{abstract}
A probe for near-field scanning optical microscopy is demonstrated based on a high index glass sphere attached to the end of a conventional atomic force microscopy tip. The sphere is machined into a pyramid geometry using a focused ion beam (FIB) instrument, coated with aluminum to confine the excitation light, and milled further with the FIB to open an aperture at the end of the tip. Near-field fluorescence images of $50 \mathrm{~nm}$ fluorescent latex spheres reveal subdiffraction limit spatial resolution, illustrating the utility of these probes for near-field scanning optical microscopy. (C) 2001 American Institute of Physics. [DOI: 10.1063/1.1430028]
\end{abstract}

Near-field scanning optical microscopy (NSOM) is a scanning probe technique that enables high resolution optical measurements to be conducted with a spatial resolution beyond the classical diffraction limit. ${ }^{1-3}$ While the technique can be implemented in several configurations, the most popular utilizes a metal-coated, tapered fiber optic probe to deliver light to nanometric dimensions. Introduced by Betzig et al. in $1991,{ }^{2}$ these metal-coated fiber optic probes have been successfully utilized to study single molecules, thin films, and in limited cases, biological samples. ${ }^{1,3}$ For the latter, the high spring constant of the fiber optic probes has generally limited the application of NSOM to biological samples that have been chemically fixed.

While the fiber optic NSOM probes have been highly successful, like all scanning probe techniques it is widely recognized that future progress in NSOM performance depends on continued advances in tip design. This has led to many reports exploring modifications to the existing fiber optic design or departures from it all together. The latter, for example, include the introduction of high resolution measurements based on energy transfer ${ }^{4}$ and the ongoing research revolving around the use of sharpened metal probes in the so-called apertureless NSOM design. ${ }^{3,5,6}$

Recently, a particularly promising approach in NSOM tip design has been demonstrated by Eckert et al. using a modified atomic force microscopy (AFM) probe. ${ }^{7}$ A solid quartz tip was grown on the end of an AFM cantilever and coated with $60 \mathrm{~nm}$ of aluminum. Without forming an aperture in the tip, the probe was utilized in constant height mode to image the emission from single molecules embedded in PMMA with $32 \mathrm{~nm}$ resolution. These results clearly demonstrate the promise of using integrated NSOM/AFM probe designs for high resolution optical imaging.

Here we report on a similar NSOM/AFM tip design that has the potential to circumvent many of the problems that currently plague the conventional fiber optic NSOM probes. The idea revolves around the use of focused ion beam (FIB) instrumentation to sculpt a small glass sphere that has been attached to the end of a conventional AFM cantilever. Once

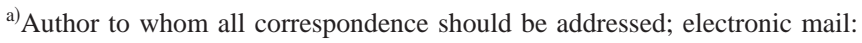
rdunn@ku.edu
}

coated with aluminum, an aperture is cut into the end of the tip using a FIB to provide a well-defined aperture for light delivery. These tips offer several advantages over the conventional fiber optic approach that include: (1) a taper angle that can be precisely controlled thus maximizing the amount of light delivered, (2) greater flexibility in using high index materials, thus reducing the ultimate resolution possible, (3)
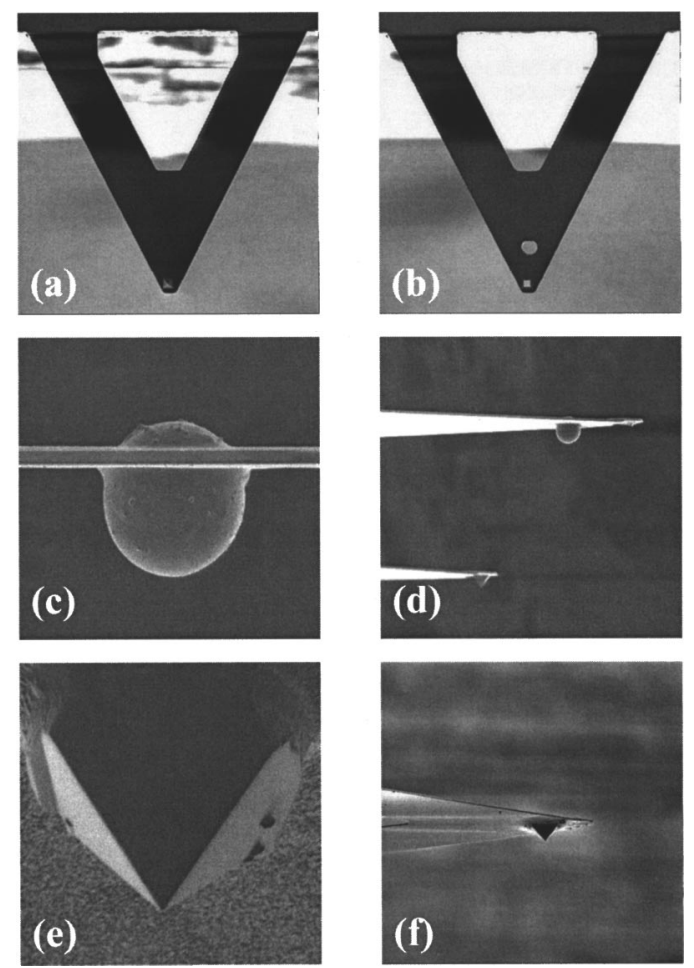

FIG. 1. Focused ion beam images of the NSOM/AFM tip fabrication process. (a) A typical silicon nitride AFM cantilever with pyramid stylus. (b) The pyramid is removed with the FIB and a $5 \mu \mathrm{m}$ hole is cut into the cantilever. (c) The hole is lightly painted with epoxy and a $7 \mu \mathrm{m}$ glass sphere is placed in the hole using standard micromanipulation techniques. (d) A larger area view of the tip shown in (c) where a conventional AFM tip with its stylus can be seen in the background. (e) The sphere is cut into a pyramid shape using the FIB. (f) The tip is coated with approximately 100 $\mathrm{nm}$ of aluminum to confine the excitation light and a small aperture is cut into the apex of the pyramid stylus with the FIB. In addition, the top side of the glass sphere is milled flat to allow efficient light coupling into the tip (not shown). 

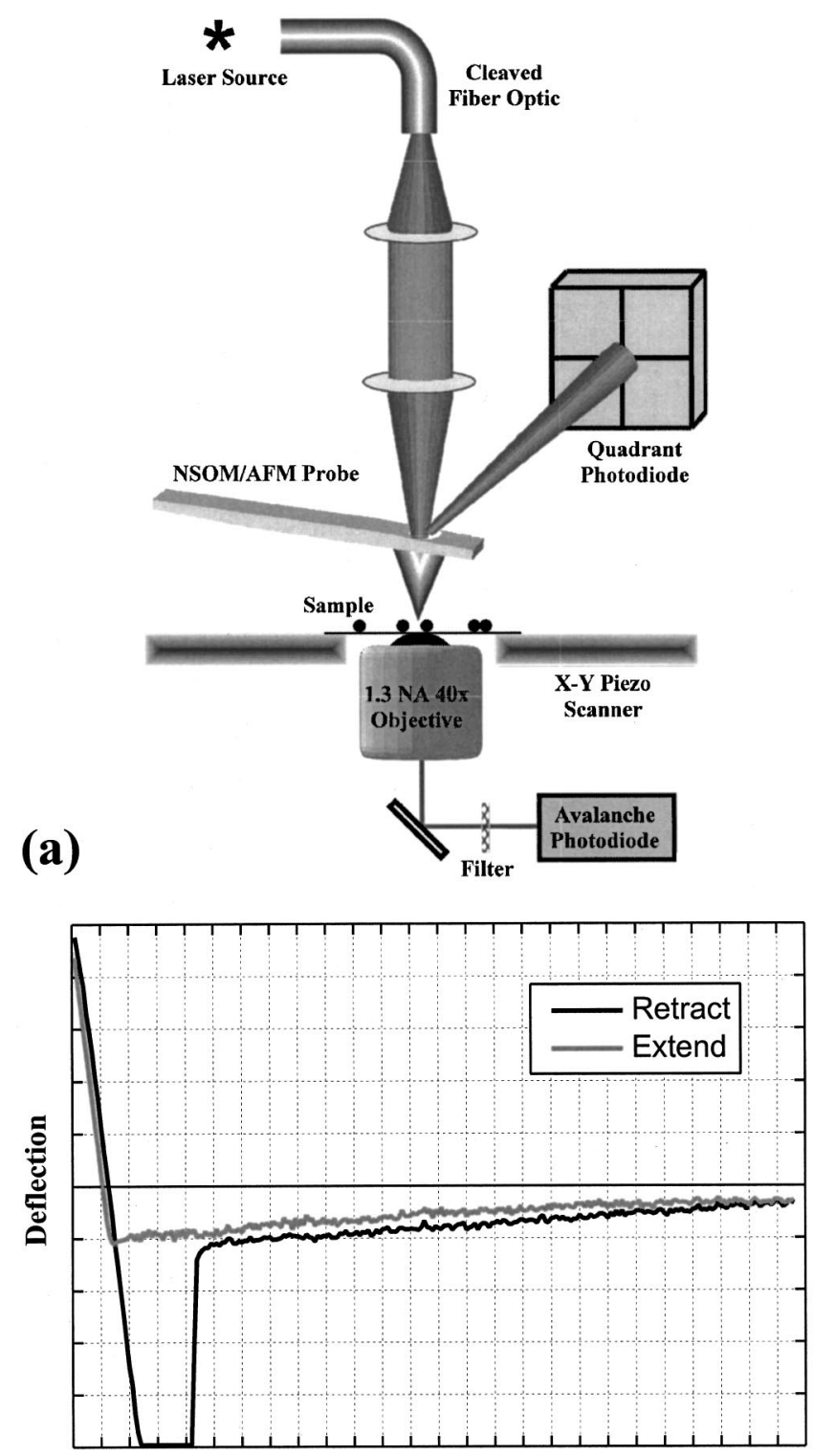

(b)

$\mathrm{Z}$ position (0.07 microns/div)

FIG. 2. (a) Schematic representation of the NSOM/AFM light path. The laser diode used for conventional AFM feedback is removed and replaced with a cleaved single mode optical fiber that delivers the desired line from an argon ion laser. This laser line serves the dual purpose of excitation and feedback source for the NSOM/AFM probe. (b) A typical approach curve for a microfabricated NSOM/AFM tip.

a tip-sample feedback control that is identical to the well understood and developed AFM techniques, and (4) a much reduced spring constant for the tip which opens the possibility of imaging viable living cells.

Figure 1 illustrates the progression in AFM tip modifications necessary to implement the NSOM tip design. The milling and high resolution imaging shown in Fig. 1 was carried out with the use of a Micrion 9000 FIB. Figure 1(a) shows a typical silicon nitride AFM tip (Digital Instruments, Model DNP-S) with a spring constant of approximately 0.12 $\mathrm{N} / \mathrm{m}$. Figure 1(b) shows the same AFM cantilever where the pyramid normally used as the stylus in AFM measurements has been removed with the FIB and a $5 \mu \mathrm{m}$ hole has been cut through the end of the cantilever. Next, the hole is painted lightly with epoxy and a $7 \mu \mathrm{m}$, high index glass sphere $(n=1.9$, MO-SCl Corporation) is placed in the hole using
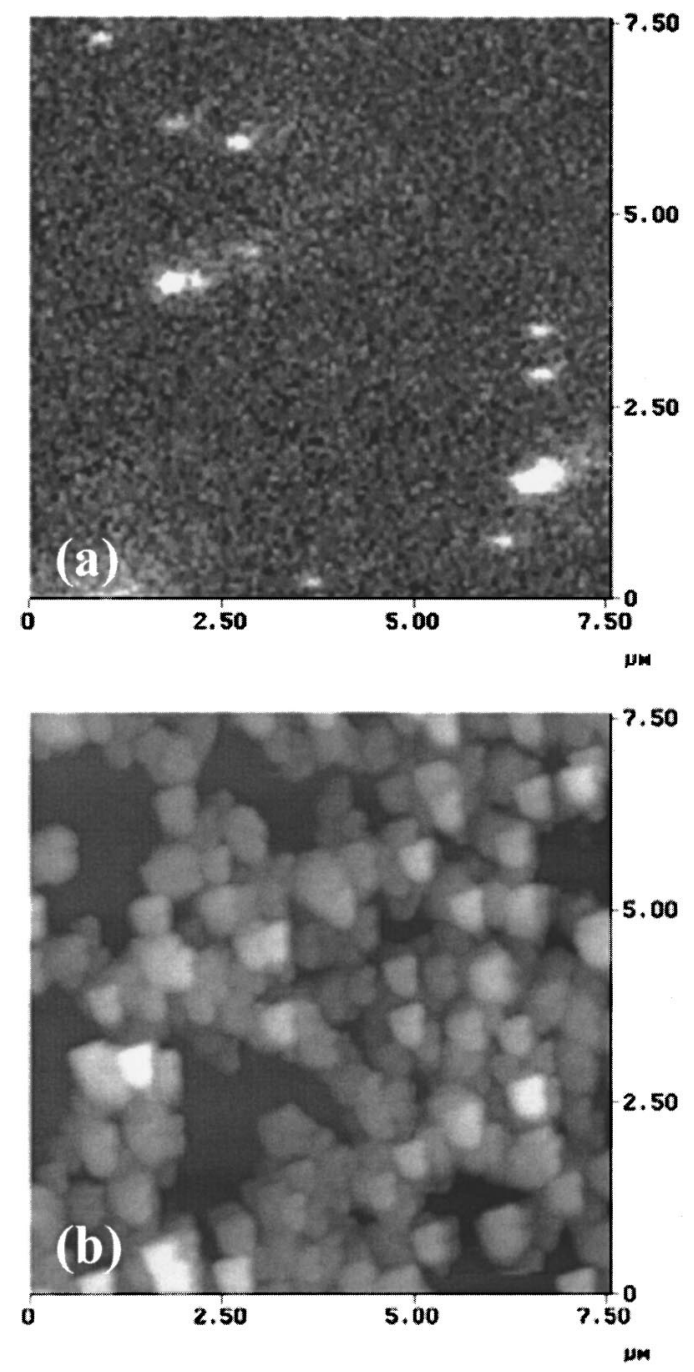

FIG. 3. (a) Near-field fluorescence and (b) force images of $50 \mathrm{~nm}$ fluorescent latex spheres embedded in an acetate matrix. The FWHM of the smallest features in the fluorescence image corresponds to $130 \mathrm{~nm}$, representing the convolution between the aperture and the $50 \mathrm{~nm}$ spheres. The resolution is currently only limited by our ability to fabricate the small aperture into the end of the stylus.

standard micromanipulation techniques. This is shown in Figs. 1(c) and 1(d).

As shown in Fig. 1(c), the glass sphere projects from both sides of the AFM cantilever, which allows FIB modification of both the light input and output sides of the sphere. The glass sphere is cut with the FIB to form a pyramid structure similar in dimensions to the pyramid stylus utilized in AFM measurements. This is illustrated in Figs. 1(e) and 1(f). It is important to stress that the use of the FIB for tip fabrication allows complete control over both the shape of the tip (pyramid, cone, etc.) and the aspect ratio, which is important for efficient light delivery. Finally, the tips are evaporatively coated with approximately $100 \mathrm{~nm}$ of aluminum and an aperture is cut into the very end of the tip using the FIB. The top of the sphere (not shown) is also milled flat with the FIB to (1) increase the coupling efficiency for light into the tip, (2) remove the aluminum coating, and (3) increase the feedback signal.

Once fabricated, the tip is mounted into a modified Dimension AFM head (Digital Instruments). The normal diode laser used for tip feedback in the AFM head has been re- 
moved and replaced with an optical fiber (Newport) that delivers the desired excitation line from an argon ion laser (514 $\mathrm{nm}$, Liconix, 5000 series). This is shown schematically in Fig. 2(a). The light coupled into the AFM head serves the dual purpose of excitation source for the NSOM tip and signal for the tip feedback loop. The dual use for the excitation light avoids many of the problems associated with eliminating stray feedback light in NSOM emission measurements.

For NSOM measurements, the AFM head is mounted in a modified Bioscope AFM (Digital Instruments) as described previously. ${ }^{8}$ Briefly, the sample is mounted in a separate $x-y$ closed-looped piezo stage (Mad City Labs, Inc.) that raster scans the sample under the tip. Emission excited by the NSOM tip is collected from below using a high numerical aperture objective (Zeiss, Fluar $40 \times 1.3 \mathrm{NA}$ ), filtered (Chroma) and imaged onto the active area of an avalanche photodiode (EG\&G, SPCM-200). Scanning and data collection are controlled with a Nanoscope IIIa (Digital Instruments) control station. All measurements were conducted in contact mode. A typical approach curve for the integrated NSOM/AFM tip is shown in Fig. 2(b), which exhibits qualitatively similar behavior to conventional AFM tip approach curves.

Figure 3 shows near-field fluorescence and force measurements taken with the tip shown in Fig. 1(e). The test sample consisted of a thin film of $50 \mathrm{~nm}$ fluorescent latex spheres (Duke Scientific Corporation) embedded in a host acetate matrix. Small fluorescent features are clearly visible in Fig. 3(a), which map the locations of the fluorescent spheres embedded in the film. These are not correlated with structural features in the force image. The full width at half maximum of the smallest fluorescent features is approximately $130 \mathrm{~nm}$, which represents the convolution between the actual tip aperture size and the size of the fluorescent spheres $(50 \mathrm{~nm})$. This resolution is comparable to that reported using conventional fiber optic NSOM probes and demonstrates the feasibility of using these tips for high reso- lution fluorescence measurements. Currently, the resolution is limited only by our ability to fabricate the small aperture in the end of the tip with the FIB, which we are currently working to improve.

While still at an early stage of development, the integrated NSOM/AFM tips reported here offer an extremely promising alternative to the fiber optic probes currently popular in NSOM applications. The integrated tips offer a highly controlled geometry, lower spring constant, greater flexibility in controlling the refractive index of the material, and better understood force imaging characteristics over the shear-force technique widely adopted in NSOM applications. These tips do suffer, however, from a more laborious fabrication process and the need for expensive FIB instrumentation, but these impediments may be minimized as the fabrication techniques are refined. Since these tips share many of the physical characteristics of AFM probes, they should be capable of imaging living cells, which has been a major obstacle in the extension of NSOM into the biological sciences.

J.R.K. gratefully acknowledges support from the NIH Dynamic Aspects of Chemical Biology predoctoral training grant. Additional support for this work was generously provided by NSF (CHE-9982052), the Alfred P. Sloan Foundation, and the Madison and Lila Self Foundation.

${ }^{1}$ R. C. Dunn, Chem. Rev. 99, 2891 (1999).

${ }^{2}$ E. Betzig, J. K. Trautman, T. D. Harris, J. S. Weiner, and R. L. Kostelak, Science 251, 1468 (1991).

3 J. Microsc. 202, (2001). Proceedings of the Sixth International Conference on Near-Field Optics and Related Techniques (NFO-6), Enschede, The Netherlands, 2000.

${ }^{4}$ S. A. Vickery and R. C. Dunn, Biophys. J. 76, 1812 (1999).

${ }^{5}$ E. J. Sanchez, L. Novotny, and X. S. Xie, Phys. Rev. Lett. 82, 4014 (1999).

${ }^{6}$ S. Kawata and Y. Inouye, Ultramicroscopy 57, 313 (1995).

${ }^{7}$ R. Eckert, J. M. Freyland, H. Gersen, H. Heinzelmann, G. Schurmann, W. Noell, U. Staufer, and N. F. De Rooij, Appl. Phys. Lett. 77, 3695 (2000).

${ }^{8}$ C. E. Talley, G. Cooksey, and R. C. Dunn, Appl. Phys. Lett. 69, 3809 (1996). 